ARTICLE

\title{
Maximizing ion accessibility in MXene-knotted carbon nanotube composite electrodes for high-rate electrochemical energy storage
}

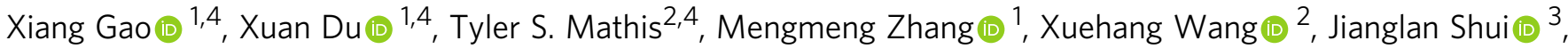 \\ Yury Gogotsi (i) ${ }^{2 \otimes} \&$ Ming Xu (i) ${ }^{1 凶}$
}

Improving the accessibility of ions in the electrodes of electrochemical energy storage devices is vital for charge storage and rate performance. In particular, the kinetics of ion transport in organic electrolytes is slow, especially at low operating temperatures. Herein, we report a new type of MXene-carbon nanotube (CNT) composite electrode that maximizes ion accessibility resulting in exceptional rate performance at low temperatures. The improved ion transport at low temperatures is made possible by breaking the conventional horizontal alignment of the two-dimensional layers of the $\mathrm{MXene} \mathrm{Ti}_{3} \mathrm{C}_{2}$ by using specially designed knotted CNTs. The large, knot-like structures in the knotted CNTs prevent the usual restacking of the $\mathrm{Ti}_{3} \mathrm{C}_{2}$ flakes and create fast ion transport pathways. The MXene-knotted CNT composite electrodes achieve high capacitance (up to $130 \mathrm{Fg}^{-1}\left(276 \mathrm{~F} \mathrm{~cm}^{-3}\right)$ ) in organic electrolytes with high capacitance retention over a wide scan rate range of $10 \mathrm{mV} \mathrm{s}^{-1}$ to $10 \mathrm{Vs}^{-1}$. This study is also the first report utilizing MXene-based supercapacitors at low temperatures (down to $-60^{\circ} \mathrm{C}$ ).

\footnotetext{
${ }^{1}$ State Key Laboratory of Materials Processing and Die \& Mold Technology, School of Materials Science and Engineering, Huazhong University of Science and Technology (HUST), 430074 Wuhan, P R China. ${ }^{2}$ A. J. Drexel Nanomaterials Institute and Department of Materials Science and Engineering, Drexel University, Philadelphia, PA 19104, USA. ${ }^{3}$ School of Materials Science and Engineering, Beihang University, 100083 Beijing, P R China. ${ }^{4}$ These authors contributed equally: Xiang Gao, Xuan Du, Tyler S. Mathis. ${ }^{凶}$ email: gogotsi@drexel.edu; ming.xu@hust.edu.cn
} 
T he storage of charge in capacitive electrochemical energy storage devices begins with the adsorption of electrolyte ions on the surface or active sites of an electrode. Only the area of the electrode that is accessible to ions can contribute to the storage of charges. Therefore, ion accessibility can influence the capacitance or capacity of the energy storage devices ${ }^{1,2}$. The influence is more obvious when the devices are operated at high rates or low temperatures, especially in the case of organic electrolytes.

Since organic electrolytes can provide a wider working voltage window than aqueous electrolytes, organic-electrolyte-based energy storage devices are more promising for achieving high energy density ${ }^{3}$. The use of organic electrolytes is also essential for the operation of energy storage devices working at sub-zero temperatures. However, organic electrolytes have larger ions and lower conductivity than aqueous electrolytes. Besides, the solvation shells of ions in organic electrolytes also have a larger size than those in aqueous electrolyte. These properties hinder charge storage and slow the ion transport kinetics of electrode materials, limiting high-rate performances, especially at low temperatures ${ }^{4-6}$. Improving ion accessibility in organic-electrolyte-based energy storage devices, such as supercapacitors, remains a challenge ${ }^{7}$.

Efforts to improve ion accessibility for high-rate performance in organic electrolytes have been addressed using two approaches: electrolyte formulation and modification of electrode structure. As an example of electrolyte tuning, Wang et al. ${ }^{8}$ investigated how organic solvents influenced the intercalation of lithium ions into porous multilayer $\mathrm{Ti}_{3} \mathrm{C}_{2} \mathrm{~T}_{x}$ and found that de-solvation had occurred in a lithium bis(trifluoromethylsulfonyl)imide/propylene carbonate (Li-TFSI/PC) electrolyte, which improved charge storage. A capacitance of $110 \mathrm{~F} \mathrm{~g}^{-1}\left(231 \mathrm{~F} \mathrm{~cm}^{-3}\right)$ at $2 \mathrm{mV} \mathrm{s}^{-1}$ was reported and the scan rate range for their MXene-based supercapacitor was from $2 \mathrm{mV} \mathrm{s}^{-1}$ to $1 \mathrm{~V} \mathrm{~s}^{-1}$ with a capacitance retention of $58 \%$. Yet only room-temperature properties were reported. Although the porous MXene used in this study facilitated the infiltration of the organic electrolytes in the electrodes at room temperature with the precise tuning of the electrolyte formulation, the low-temperature performance would still be limited because of the dramatically increased viscosity of electrolytes at low temperatures. Another study on tuning electrolyte composition showed that matching between the size of the electrode pores of carbon electrodes with the solvated ions of the electrolyte played an important role in achieving exceptional low-temperature performance ${ }^{9}$. A double-layer capacitance of $177 \mathrm{~F} \mathrm{~g}^{-1}$ at $5 \mathrm{mV} \mathrm{s}^{-1}$ was published with the rate performance from 5 to $500 \mathrm{mV} \mathrm{s}^{-1}$, while the capacitance retention of $\sim 92.6 \%$ was obtained over the temperature range of 20 to $-70^{\circ} \mathrm{C}$ at $5 \mathrm{mV} \mathrm{s}^{-1}$. However, the heteroatoms (e.g., N, S, O) of the biomassderived carbon limited the voltage window to $2.5 \mathrm{~V}$, potentially lowering the energy density. Additionally, the rate performance data implied a limited ion transport and ion accessibility associated with the electrode structure.

When attempting to modify electrode structures themselves to improve ion accessibility, the common approaches include introducing pores in the surface of an electrode material through chemical etching ${ }^{10,11}$, increasing the interlayer spacing through modification of surface chemistry, or through the intercalation of pillaring materials to prevent restacking of two-dimensional (2D) sheets $^{12-15}$. For example, Dall'Agnese et al. ${ }^{16}$ used multiwall carbon nanotubes (MWCNTs) as the spacers to separate MXene sheets and reported the capacitances ranging from $85 \mathrm{~F} \mathrm{~g}^{-1}\left(245 \mathrm{~F} \mathrm{~cm}^{-3}\right)$ at $2 \mathrm{mV} \mathrm{s}^{-1}$ to $63 \mathrm{~F} \mathrm{~g}^{-1}\left(181 \mathrm{~F} \mathrm{~cm}^{-3}\right)$ at $100 \mathrm{mV} \mathrm{s}^{-1}$. Another study reported MXene/MWCNT composite electrodes prepared by selfassembly of negatively charged, metallic $\mathrm{Ti}_{3} \mathrm{C}_{2} \mathrm{~T}_{x}$ MXene flakes and positively charged CNTs as spacers. The capacitance of $75 \mathrm{Fg}^{-1}$ $\left(175 \mathrm{~F} \mathrm{~cm}^{-3}\right)$ at $10 \mathrm{mV} \mathrm{s}^{-1}$ was achieved, with the rate performance from 0.5 to $100 \mathrm{mV} \mathrm{s}^{-1}$ (ref. ${ }^{13}$ ). Although the MXene/
MWCNT composite electrodes produced by these approaches appear to have a larger interlayer spacing for their improved ion accessibility compared to unmodified MXenes, the scanning rate has never been achieved higher than $100 \mathrm{mV} \mathrm{s}^{-1}$ and only roomtemperature properties have been reported. Even though these methods increase the spacing between the layers of the $2 \mathrm{D}$ electrode materials (e.g. MXene), the stacking problem persists that the $2 \mathrm{D}$ layers remain horizontally stacked. Therefore, the ion transport in the electrode is very tortuous and slow in the direction normal to the electrode surface, which would be an obstacle for high-rate performance and low-temperature operation. Recently, Sun et al. ${ }^{17}$ fabricated a graphene $/ \mathrm{Nb}_{2} \mathrm{O}_{5}$ composite electrode by using a three-dimensional (3D) graphene framework as a conductive scaffold that resulted in high rate energy storage with a capacity retention of $\sim 40 \%$ over a current density range of 0.2 to $20 \mathrm{~A} \mathrm{~g}^{-1}$. Although the $\mathrm{Nb}_{2} \mathrm{O}_{5}$ was not modified, i.e. the ion intercalation tunnels remained narrow and the electrical conductivity was low, the 3D graphene framework provided fast ion transport, leading to improved high-rate performance. As these studies show, in order to further improve the performance of energy storage systems at high rates, consideration must be given to both ion accessibility and ion transport.

In this study, we developed an approach to achieve high-rate performance in an organic electrolyte through the construction of a 3D electrolyte-accessible electrode structure. $\mathrm{Ti}_{3} \mathrm{C}_{2} \mathrm{~T}_{x}$ (for simplicity we will write the chemical formula as $\mathrm{Ti}_{3} \mathrm{C}_{2}$ throughout the remainder of this paper) was chosen to form an interconnected network where the specially synthesized knotted CNTs were encased as the support for the $\mathrm{Ti}_{3} \mathrm{C}_{2}$ network. To simultaneously maximize ion accessibility and minimize the tortuosity of ion transport pathways, the structure of the electrodes was modified from being heavily stacked to being highly misaligned by optimizing the sizes and the mass ratios of the knotted CNTs and $\mathrm{Ti}_{3} \mathrm{C}_{2}$ flakes. The MXene-knotted CNT composite electrodes exhibited a high capacitance of $130 \mathrm{~F} \mathrm{~g}^{-1}\left(276 \mathrm{~F} \mathrm{~cm}^{-3}\right)$ with a capacitance retention of $\sim 56 \%$ over three orders of magnitude of scan rates from $10 \mathrm{mV} \mathrm{s}^{-1}$ to $10 \mathrm{~V} \mathrm{~s}^{-1}$. They also demonstrated superior stability in organic electrolytes without any capacitance loss upon 10,000 cycles. Our new electrodes even enabled impressive low-temperature operation. A high capacitance retention was obtained over the temperature range of 20 to $-60^{\circ} \mathrm{C}$ at $20 \mathrm{mV} \mathrm{s}^{-1}$. At $-30{ }^{\circ} \mathrm{C}$, the asymmetric device was capable of operating with a $4.2 \mathrm{~V}$ voltage window without any parasitic reactions or losses in capacitive behavior. It is the largest operating voltage window that has been reported so far for a MXene-based device. An energy density of $59 \mathrm{Wh} \mathrm{kg}^{-1}$ and a power density of $9.6 \mathrm{~kW} \mathrm{~kg}^{-1}$ were obtained at $-30{ }^{\circ} \mathrm{C}$, surpassing the best values reported for supercapacitors with $2 \mathrm{D}$ electrode materials operating at low temperatures.

\section{Results}

Design of MXene-knotted CNT composite electrodes. As discussed in the previous section, the mixing of nanomaterials (e.g. single- or multiwall CNTs, metal oxides) with MXenes has already been used to modify the interlayer spacing of MXene; however, the restacking of the MXene flakes still leads to a planar, paper-like electrode architecture with an in-plane orientation of the $2 \mathrm{D}$ sheets, severely limiting the accessibility of the electrolyte (Supplementary Fig. 1), which hinders the pseudocapacitive performance of MXenes at high scan rates in aqueous electrolyte and results in poor rate performance in organic electrolytes ${ }^{18-21}$.

To create the electrodes with a 3D electrolyte-accessible architecture, a special type of CNTs with large, knot-like structures with the size tens to hundreds of times larger than the typical nanomaterials that were used as spacer molecules were 
a
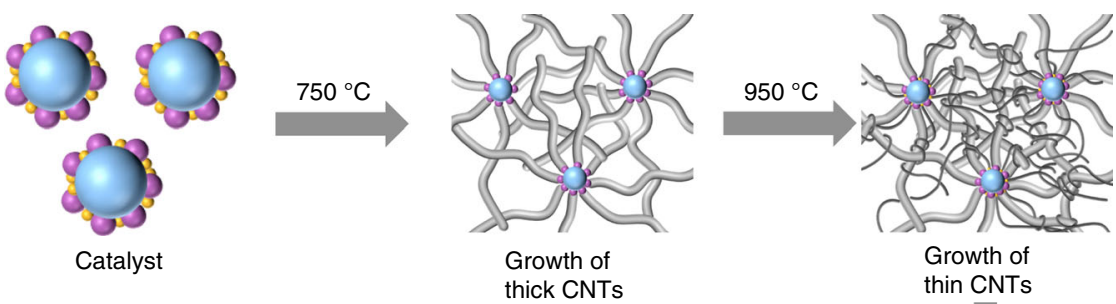

b

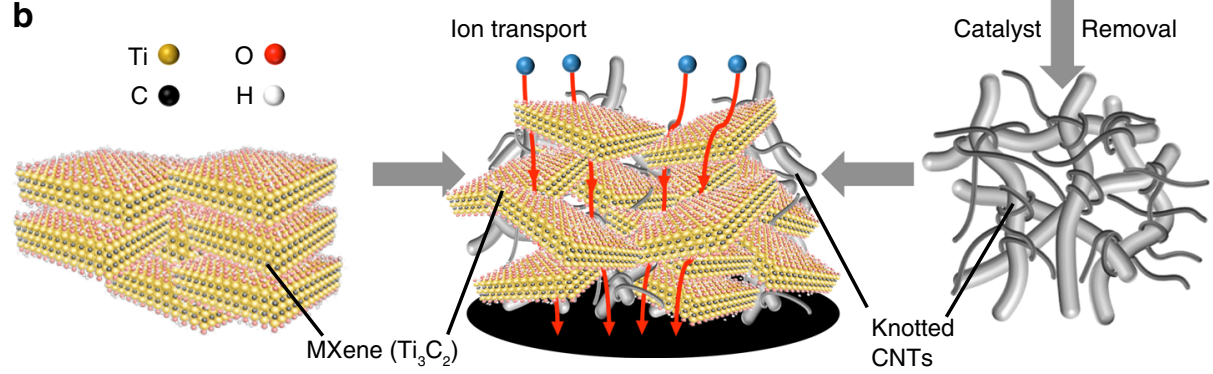

Fig. 1 Design of the MXene-knotted CNT composite electrodes for efficient ion transportation. a Schematic of the synthesis process for the knotted CNTs, where thin CNTs were intertwined with thicker CNTs. b Schematic of a MXene-knotted CNT composite electrode with reduced horizontal orientation and lower ion transport tortuosity through the bulk of the electrode.

synthesized in order to misalign the $\mathrm{Ti}_{3} \mathrm{C}_{2}$ flakes in the electrode structure. We will refer to this special type of CNTs by calling them knotted CNTs. The knotted CNTs were produced by growing thick and thin CNTs in one assembly where the flexible thin CNTs were intertwined with stiff thick CNTs to form large knots. It should be noted that these large knots were not able to be produced by using CNTs with identical dimeters ${ }^{13,22}$. The thick CNTs were too stiff to form knots, and while the flexible thin CNTs can form knot-like structures, the size of the knots were not large enough to break the stacking of $\mathrm{Ti}_{3} \mathrm{C}_{2}$ flakes.

In order to simultaneously grow CNTs of different sizes, the catalyst nanoparticles with the catalysts of two different sizes were specially designed to facilitate the growth of the knotted CNTs (Fig. 1a). The catalyst nanoparticles were placed in a temperatureshifting two-stage fluidized bed reactor with separate temperature zones where the smaller catalyst produced thinner CNTs and the larger catalyst facilitated the growth of thick CNTs in the specific temperature zones (Supplementary Figs. 2 and 3 and Methods). Compared with the water-assisted chemical vapor deposition (CVD) method ${ }^{23-26}$, the knotted CNTs synthesized using the fluidized bed reactor had significant amounts of residual catalyst which needed to be removed via a purification possess. The MXeneknotted CNT composite electrodes were then prepared through a self-assembly process where $\mathrm{Ti}_{3} \mathrm{C}_{2}$ suspensions were mixed with knotted CNT solution before being filtered using vacuum assisted filtration to form freestanding films. A range of films with CNT contents (i.e. the mass ratio of CNTs to the electrode) from 5 to $34 \%$ were made and studied (Supplementary Table 1 and Supplementary Fig. 4). The thicknesses of the electrodes were controlled to be approximately $10 \mu \mathrm{m}$ and all the electrodes displayed a mass loading higher than $1.8 \mathrm{mg} \mathrm{cm}^{-2}$. Detailed descriptions of the synthesis of the knotted CNTs and the electrode fabrication process are available in the 'Methods' section.

Because of the mechanical constraints of the knotted CNTs, the large knotted structures were capable of maintaining the structural integrity of the electrode film while preventing the restacking of the MXene flakes during the electrode fabrication process, which has not been demonstrated previously (Fig. 1b). The structure of these composite electrodes ensures efficient ion transportation by dramatically reducing the tortuosity of the ion transport pathways.
The successful synthesis of the knotted CNTs and the fabrication of the MXene-knotted CNT composite electrodes were confirmed by scanning electron microscopy (SEM) and transmission electron microscopy (TEM). Figure $2 \mathrm{a}$ showed the structure of the knotted CNTs, where the thin flexible CNTs $(\varnothing=15 \pm 8 \mathrm{~nm}$; length $=0.2-1 \mu \mathrm{m})$ were wound around the thick CNTs $(\varnothing=55 \pm 5 \mathrm{~nm}$; length $=5-15 \mu \mathrm{m})$, effectively forming large knots with sizes upwards of $200 \pm 20 \mathrm{~nm}$. To investigate their mechanical stability the knotted CNTs were sonicated at $500 \mathrm{~W}$ for $50 \mathrm{~min}$. TEM images of the sonicated CNTs revealed that the cohesive structure of the CNT knots remained after sonication without a significant reduction in the size of the knots (Fig. 2b).

The breaking of the alignment of the MXene flakes by the knotted CNTs can be seen by looking at SEM images of the tops and cross-sections of the different MXene-knotted CNT composite electrodes (Fig. 2c, d). Figure $2 \mathrm{c}$ showed the porous morphology of the surface of the MXene-knotted CNT composite electrodes where several vertically oriented $\mathrm{Ti}_{3} \mathrm{C}_{2}$ flakes can be seen. The cross-sectional images showed how the MXene-knotted CNT composite electrodes had a significantly lower degree of horizontal orientation relative to the pure MXene film (Fig. 2d). In addition, the X-ray diffraction (XRD) patterns of the MXeneknotted CNT composite electrodes (Supplementary Fig. 5) showed that the orientation of the $\mathrm{Ti}_{3} \mathrm{C}_{2}$ flakes in the MXeneknotted CNT composite electrodes was more random since only the (002) peak was observable. None of the higher order $(00 \ell)$ peaks that were characteristic of freestanding MXene films with highly aligned flakes were present. The (002) peaks were located at $8.19^{\circ}, 6.03^{\circ}, 5.61^{\circ}, 5.22^{\circ}$, and $4.74^{\circ}$ for the samples with CNT contents from $0 \%$ to $34 \%$, respectively. The corresponding interlayer spacings calculated using the Bragg equation were $10.8,14.7,15.8,16.9$, and $18.6 \AA^{27,28}$. Higher magnification SEM images (Fig. 2e) showed that the CNT knots were confined within the spaces between $\mathrm{Ti}_{3} \mathrm{C}_{2}$ flakes. The entirety of the electrode structure was composed of CNT knots encased by $\mathrm{Ti}_{3} \mathrm{C}_{2}$ flakes, exhibiting a 3D architecture with both increased interlayer spacing and misaligned MXene flakes. Compared to 2D stacked electrode structures that only had enlarged interlayer spacing, our 3D electrode structure provided increased open space for enhanced accessibility and reduced the tortuosity of the ion 

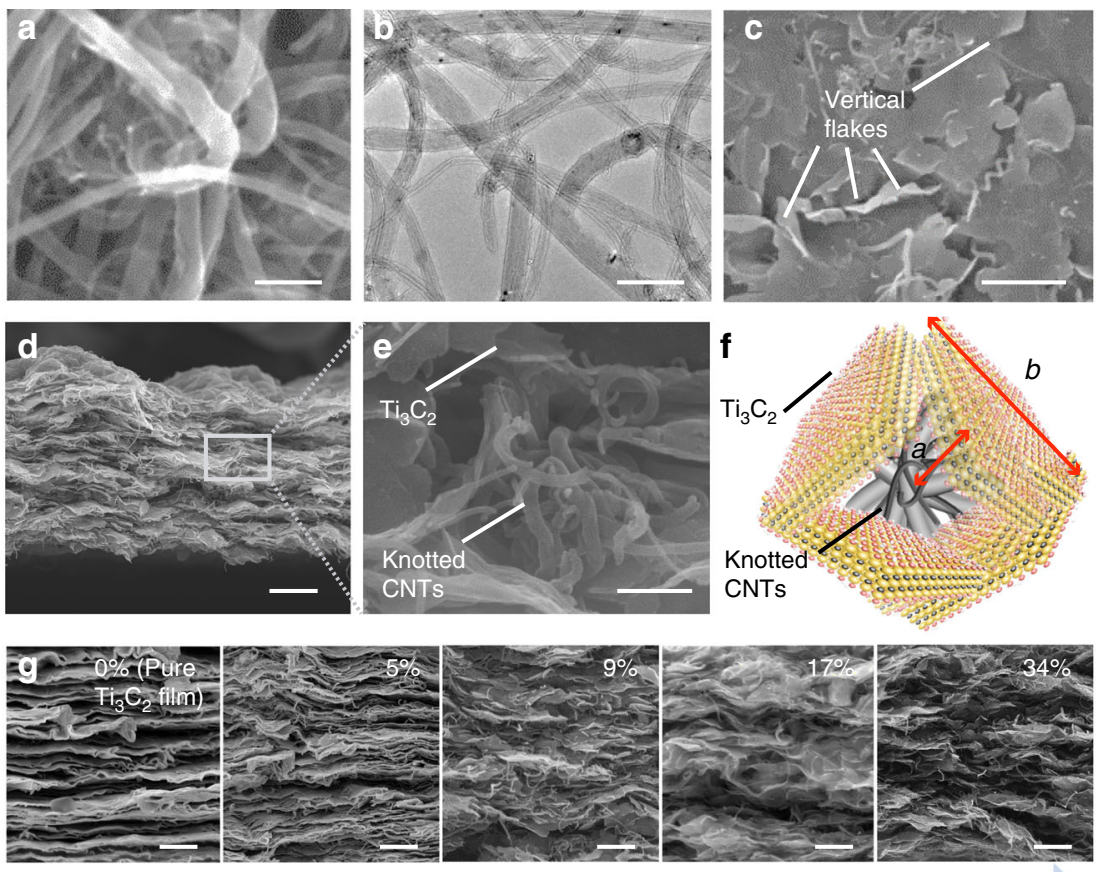

Increased CNT content (horizontal alignment decreases)

Fig. 2 Characterization of the knotted CNTs and MXene-knotted CNT composite electrodes. a SEM and $\mathbf{b}$ TEM images of the knotted CNTs. $\mathbf{c}$ Top view and $\mathbf{d}$ cross-sectional SEM images of an MXene-knotted CNT composite electrode with $17 \%$ CNTs. e SEM image and $\mathbf{f}$ corresponding schematic of how the alignment of the $\mathrm{Ti}_{3} \mathrm{C}_{2}$ flakes was broken by the knotted CNTs to form 3D networked structure. " $a$ " and " $b$ " ( $1: 2$ ratio) in the schematic correspond to the radius of the knots (taking the knots as circles) and the size of the $\mathrm{Ti}_{3} \mathrm{C}_{2}$ flakes, respectively. $\mathbf{g}$ Cross-sectional SEM images of $\mathrm{MXene-knotted} \mathrm{CNT}$ composite electrodes with different CNT contents from $0 \%$ (pure $\mathrm{Ti}_{3} \mathrm{C}_{2}$ film) to $34 \%$. Scale bars, $100 \mathrm{~nm}(\mathbf{a}, \mathbf{b}), 1 \mu \mathrm{m}(\mathbf{c}, \mathbf{g}), 2 \mu \mathrm{m}(\mathbf{d}), 200 \mathrm{~nm}(\mathbf{e})$

transport pathways to improve the transport of charge storing ions through the electrode.

It is important to note that correctly matching the sizes of the CNT knots and the $\mathrm{Ti}_{3} \mathrm{C}_{2}$ flakes is highly important for the successful formation of the $3 \mathrm{D}$ electrolyte-accessible architecture. Figure $2 \mathrm{f}$ showed a schematic for a small region of the MXeneknotted CNT composite electrode structure that illustrated how the size of the $\mathrm{Ti}_{3} \mathrm{C}_{2}$ flakes must be slightly larger than the CNT knots, ensuring that the misaligned $\mathrm{Ti}_{3} \mathrm{C}_{2}$ flakes form a continuous network with ample amounts of interstitial spaces. To achieve this structure (Fig. 2e, f), CNT knots were synthesized to be $200 \pm 20 \mathrm{~nm}$ in size, while the $\mathrm{Ti}_{3} \mathrm{C}_{2}$ flakes that were used had an average flake size of $\sim 250 \mathrm{~nm}$ (Supplementary Fig. 6). Smaller CNT knots were not capable of breaking the alignment of the $\mathrm{Ti}_{3} \mathrm{C}_{2}$ flakes while CNT knots that were too large size damaged the conductive network of the MXene flakes (Supplementary Fig. 7), resulting in the electrode having poor electrical conductivity.

The CNT content also plays a role in the formation of the $3 \mathrm{D}$ electrolyte-accessible architecture. As shown in Fig. 2g, increasing the knotted CNT content resulted in the architecture of the electrodes transitioning from a stacked, planar structure to a $3 \mathrm{D}$ networked architecture. When the CNT content was small (5\%), negligible variations in the electrode structure occurred, and the cross-sectional image of the 5\% CNT electrode showed that the film was still highly aligned. Significant variations were only observed when the CNT content was increased to 9\%. However, the 9\% CNT electrode still had a relatively densely packed structure with some regions of stacked MXene flakes visible. The structure of the $17 \%$ CNT electrode was noticeably less packed, while the cross-sectional image of the $34 \% \mathrm{CNT}$ electrode showed there were still some regions of densely stacked $\mathrm{Ti}_{3} \mathrm{C}_{2}$. Incorporating higher amounts of knotted CNTs likely led to the separation of the MXene and CNT phases within the electrode structure due to the significant difference in the densities of the CNTs and $\mathrm{Ti}_{3} \mathrm{C}_{2}$, which would affect the conductivity of the electrodes. The sheet resistance of the different MXene-knotted CNT composite films decreased from 0.15 to $10 \Omega$ per square as the CNT content increased from $0 \%$ to $34 \%$ (Supplementary Table 1).

High-rate electrochemical performance in an organic electrolyte. To investigate the electrochemical performance of the MXene-knotted CNT composite electrodes, we first evaluated the electrodes in $1 \mathrm{M}$ 1-ethyl-3-methylimidazolium bis(trifluoromethylsulfonyl)imide/ acetonitrile (EMIM-TFSI/ACN). Although the electrolyte contained a large cation (EMIM), the MXene-knotted CNT composite electrodes showed a capacitance of $63 \mathrm{Fg}^{-1}$ at $10 \mathrm{mV} \mathrm{s}^{-1}$ and good rate performance with a capacitance retention of $56 \%$ from $10 \mathrm{mV} \mathrm{s}^{-1}$ to $2 \mathrm{~V} \mathrm{~s}^{-1}$ (Supplementary Fig. 8a, b). To further improve the capacitance, lithium bis(trifluoromethylsulfonyl)imide (Li-TFSI) was mixed with the EMIM-TFSI/ACN (EMIM-TFSI: Li-TFSI $=1: 1$ ) as the Li ions can intercalate into the $\mathrm{Ti}_{3} \mathrm{C}_{2}$ resulting in increased charge storage. Furthermore, the dissimilar cations in the electrolyte were also beneficial for decreasing the freezing point of the electrolyte to improve low-temperature operation ${ }^{29,30}$. The cyclic voltammograms (CVs) at different potential windows were tested at $10 \mathrm{mV} \mathrm{s}^{-1}$ and displayed a sharp irreversible peak at the potential lower than $-1.5 \mathrm{~V}$ versus Ag (Supplementary Fig. 8c). Therefore, the working potential window in the mixed electrolyte was finally chosen to be -1.5 to $0.3 \mathrm{~V}$. Figure $3 \mathrm{a}, \mathrm{b}$ showed the rate performance of the MXene-knotted CNT composite electrodes with different $\mathrm{CNT}$ contents ranging from $0 \%$ (pure $\mathrm{Ti}_{3} \mathrm{C}_{2}$ film) to $34 \%$ in the mixed electrolyte (EMIM-TFSI/LiTFSI/ACN). While the pure $\mathrm{Ti}_{3} \mathrm{C}_{2}$ electrode $(0 \% \mathrm{CNTs})$ had a 

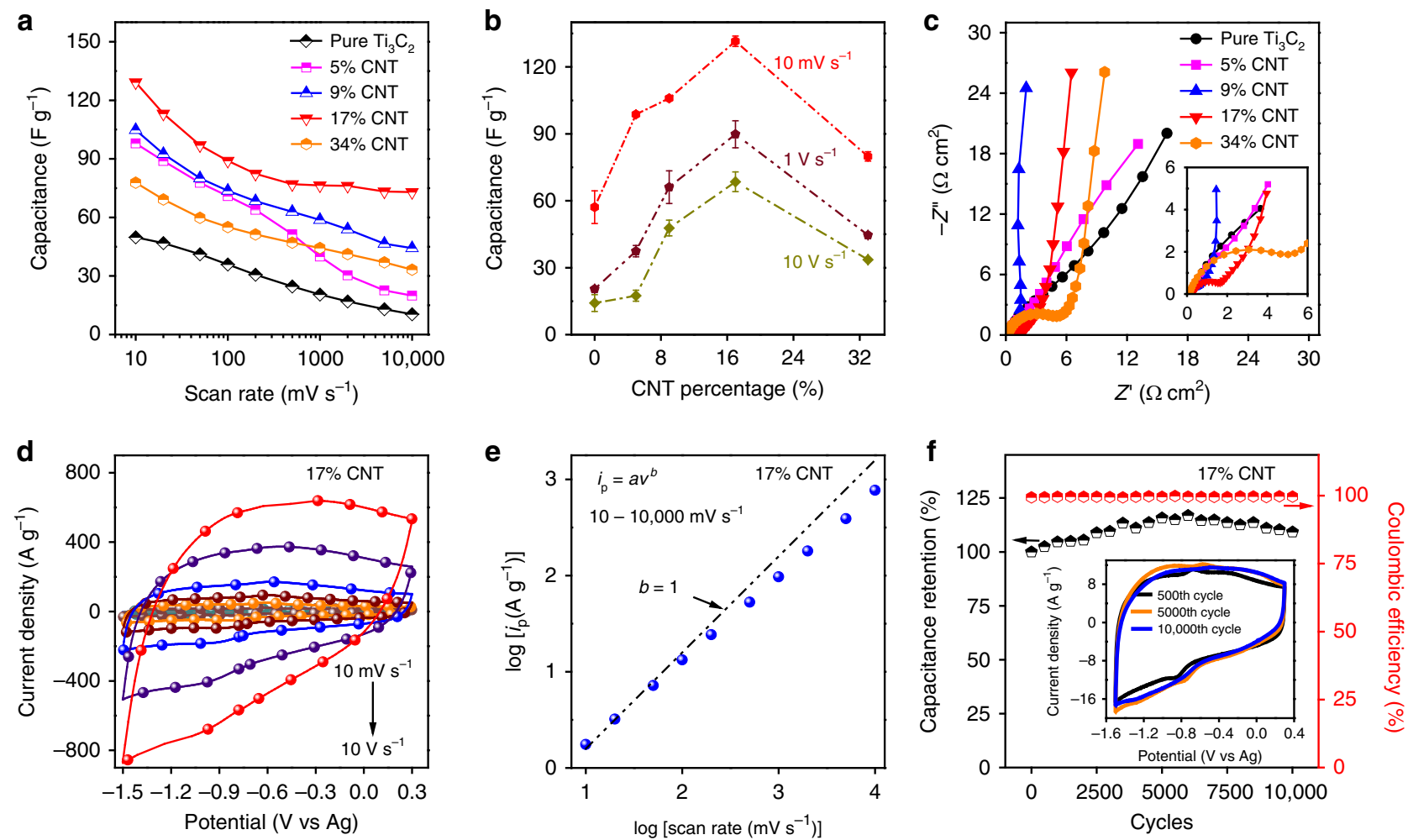

Fig. 3 Electrochemical analysis of the MXene-knotted CNT composite electrodes in an organic mixed electrolyte (EMIM-TFSI: Li-TFSI = 1:1). a Rate performance of the electrodes with different CNT contents from $0 \%$ (pure $\mathrm{Ti}_{3} \mathrm{C}_{2}$ ). $\mathbf{b}$ Capacitance of the electrodes as a function of CNT content at $10 \mathrm{mV} \mathrm{s}^{-1}, 1 \mathrm{Vs}^{-1}$, and $10 \mathrm{Vs}^{-1}$. The error bars represent the standard deviation. c Electrochemical impedance spectroscopy (EIS) data for the different electrodes taken at $\mathrm{O} V$ versus open circuit voltage. The inset showed a magnified view of the high-frequency region. $\mathbf{d}$ Cyclic voltammograms at different scan rates, e plot of the anodic peak current versus the scan rate, and $\mathbf{f}$ long-term cycling performance and corresponding Coulombic efficiency at $100 \mathrm{mV} \mathrm{s}^{-1}$ for the $17 \%$ CNT electrode.

specific capacitance of $50 \mathrm{Fg}^{-1}$ at $10 \mathrm{mV} \mathrm{s}^{-1}$ and a capacitance retention of only $20 \%$ from $10 \mathrm{mV} \mathrm{s}^{-1}$ to $10 \mathrm{~V} \mathrm{~s}^{-1}$, the MXeneknotted CNT composite electrode with $17 \%$ CNTs had significantly improved specific capacitance and rate performance with $\sim 130 \mathrm{Fg}^{-1}$ at $10 \mathrm{mV} \mathrm{s}^{-1}$ and $\sim 73 \mathrm{Fg}^{-1}$ at $10 \mathrm{~V} \mathrm{~s}^{-1}$, approximately $56 \%$ of the initial capacitance value at $10 \mathrm{mV} \mathrm{s}^{-1}$, surpassing most of the best values that have been reported for the MXene-based electrodes tested in organic electrolytes ${ }^{12,15,16}$. In all, $130 \mathrm{~F} \mathrm{~g}^{-1}\left(\sim 276 \mathrm{~F} \mathrm{~cm}^{-3}\right)$ is also the highest reported capacitance for $\mathrm{Ti}_{3} \mathrm{C}_{2}$ MXene-based electrodes for electrolytes using acetonitrile as the solvent (Supplementary Fig. 9) (16, $^{8,16}$.

The increased rate performance with the increased CNT content up to $17 \%$ was attributed to a transition of the structure of the electrode from a stacked, planar structure to a 3D networked architecture, as described previously. The decreases in capacitance and capacitance retention occurred when the CNT content was increased to $34 \%$, which was likely due to the larger internal resistance of the $34 \%$ CNT electrode caused by the discontinuous MXene network (Fig. $2 \mathrm{~g}$ and Supplementary Table 1). Moreover, we prepared a composite electrode by mixing MXene with commercially available non-knotted MWCNTs for comparison (Supplementary Fig. 10). As shown by Supplementary Fig. 10a, b, the non-knotted MWCNTs possessed a uniform diameter of $\sim 35 \mathrm{~nm}$ and the electrode displayed a $2 \mathrm{D}$ stacked structure, which was very different from the $3 \mathrm{D}$ electrolyte-accessible electrodes structure formed by our knotted CNTs. The MXene/non-knotted MWCNT electrode only showed a capacitance retention of $39 \%$ from 10 to $500 \mathrm{mV} \mathrm{s}^{-1}$ (Supplementary Fig. 10c, d). The results indicated that the ion transport in the MXene/non-knotted MWCNT electrode remained tortuous and the increases in ion accessibility were still limited even when the interlayer spacing of the electrode was increased compared to the pure MXene. It is clear that the construction of a 3D electrolyte-accessible electrode structure through the use of specially synthesized knotted CNTs has a significant effect on both reducing the tortuosity of ion transport and increasing ion accessibility.

Electrochemical impedance spectroscopy (EIS) measurements were performed using each of the MXene-knotted CNT composite electrodes and the pure MXene electrode at open circuit voltage (OCV). As shown in Fig. 3c, the resulting Nyquist plots for the MXene-knotted CNT composite electrodes with the CNT contents higher than $9 \%$ showed significant changes in the low-frequency region relative to the MXene pure film, with each electrode showing nearly vertical lines normal to the real axis $\left(Z^{\prime}\right)$, which was consistent with the improved ion accessibility and transport in the electrodes with higher knotted CNT loading ${ }^{31,32}$. In contrast, the Nyquist plots of the pure $\mathrm{Ti}_{3} \mathrm{C}_{2}$ MXene electrode and the 5\% CNT electrode both showed a nearly constant increase in the imaginary and real impedance, indicating the sluggish ion diffusion for these densely packed electrodes. The evolution of the Nyquist plots revealed how breaking the stacking of the $\mathrm{Ti}_{3} \mathrm{C}_{2}$ flakes was necessary for improving the electrochemical kinetics.

A full electrochemical evaluation was conducted to further evaluate the full capabilities of the $17 \%$ CNT electrode as the best performing MXene-knotted CNT composite electrode. Figure 3d showed CVs for the $17 \% \mathrm{CNT}$ electrode from $10 \mathrm{mV} \mathrm{s}^{-1}$ to $10 \mathrm{~V} \mathrm{~s}^{-1}$. In the plot of capacitance versus potential derived from cyclic CVs (Supplementary Fig. 8d), two de-intercalation peaks can be seen at -0.45 and $-0.62 \mathrm{~V}$ versus $\mathrm{Ag}$ in the anodic scan. The two peaks may 
correspond to the de-intercalation processes for the EMIM and Li ions $^{8,16}$. The corresponding cathodic peaks showed small peak separation, indicating that the charge storage process had a fast, non-diffusion-limited intercalation mechanism ${ }^{33,34}$. The coulombic efficiency increased from 94.4 to $99.4 \%$ at the scan rate increasing from 10 to $200 \mathrm{mV} \mathrm{s}^{-1}$ and maintained at $\sim 100 \%$ at the high scan rate from 200 to $10,000 \mathrm{mV} \mathrm{s}^{-1}\left(10 \mathrm{~V} \mathrm{~s}^{-1}\right)$ (Supplementary Fig. 8e). Additionally, the X-ray photoelectron spectroscopy (XPS) spectra of the MXene-knotted CNT electrodes before and after cycling showed that the surface chemistry was not noticeably altered, indicating that the electrochemical reactions did not significantly affect the surface chemistry of the MXene in this system (Supplementary Fig. 11). These results were consistent with the nonlinear curves of the galvanostatic charge-discharge (GCD) tests shown in Supplementary Fig. 12 and the impedance spectra recorded at different applied potentials (Supplementary Fig. 13). As shown in Fig. 3e, the analysis of the dependence of the peak current on the scan rate indicated that the $b$ value of the $17 \% \mathrm{CNT}$ electrode was close to 1 over a large scan rate range of 10 to $10,000 \mathrm{mV} \mathrm{s}^{-1}$, which was characteristic of non-diffusion limited pseudocapacitive charge storage $^{8}$. Long-term cycling stability of the same electrode tested at $100 \mathrm{mV} \mathrm{s}^{-1}$ showed that the MXene-knotted CNT composite electrode was highly stable with almost no capacitance decay observable after 10,000 cycles (Fig. 3f) with a consistent coulombic efficiency near $\sim 100 \%$. As addressed above, the structural stability of the knotted CNTs can even withstand sonication due to the mechanical constraints (Fig. 2b). The knotted CNTs were capable of maintaining the structural integrity of the electrode film by preventing the restacking of the MXene flakes upon charge/ discharge, which guaranteed the efficient ion transportation during long-term cycling.

Low-temperature operation of full cell devices. Electrochemical energy storage at low temperatures is challenging due to the reduced mobility and transport of electrolytes near their freezing point. Using a highly conductive electrode material like $\mathrm{Ti}_{3} \mathrm{C}_{2}$ MXene with improved electrolyte accessibility can potentially mitigate the negative effects of lower temperatures. Since MXene would be oxidized at positive potentials and cannot be used as a positive electrode, we assembled asymmetric full cells with a MXene-knotted CNT composite electrode as the negative electrode and an aligned CNT electrode as the positive electrode to evaluate the performance of the MXene-knotted CNT composite electrodes at low temperatures (Fig. $4 \mathrm{a}-\mathrm{c}$ and Supplementary Figs. 14 and 15). The aligned CNT electrode showed a working potential of $1.5 \mathrm{~V}$ as the positive electrode (Fig. $4 \mathrm{a}$ ). The voltage window of the cell was maximized by balancing the mass ratio between the positive and negative electrodes. A voltage window of $3 \mathrm{~V}$ was realized with a mass ratio between the positive and negative electrodes of $\sim 3: 2$. The capacitance of the aligned CNT electrode $\left(94 \mathrm{~F} \mathrm{~g}^{-1}\right.$ at $\left.10 \mathrm{mV} \mathrm{s}^{-1}\right)$ was found to be lower than that of the MXene-knotted CNT composite electrode $\left(\sim 130 \mathrm{Fg}^{-1}\right.$ at $10 \mathrm{mV} \mathrm{s}^{-1}$ ) (Supplementary Fig. 14). Since the capacitance of the cell was limited by the lower-capacitance electrode, the aggregate capacitance can be further improved by using the positive electrode possessing higher capacitance. The CVs showed that a set of reversible redox peaks were still present in the full cell with negligible decreases in the capacitance occurring when the scan rate increased from 50 to $500 \mathrm{mV} \mathrm{s}^{-1}$ (Fig. $4 \mathrm{~b}$ and Supplementary Fig. 15a). The GCD curves in Fig. 4c were symmetric with no plateau from 1 to $20 \mathrm{Ag}^{-1}$, indicating the pseudocapacitive mechanism. Moreover, the cell also showed good stability with a capacitance retention of $90 \%$ after 8000 cycles (Supplementary Fig. 16).
Figure $4 \mathrm{~d}$ showed the $\mathrm{CV}$ curves of the asymmetric cell with a $17 \% \mathrm{CNT}$ electrode as the negative electrode at $20 \mathrm{mV} \mathrm{s}^{-1}$ from 20 to $-60{ }^{\circ} \mathrm{C}$. At $-10^{\circ} \mathrm{C}$ the asymmetric cell had a capacitance of $\sim 20 \mathrm{~F} \mathrm{~g}^{-1}$, and approximately $50 \%$ of the cell capacitance (relative to $20^{\circ} \mathrm{C}$ ) was retained at $-50{ }^{\circ} \mathrm{C}$ with the CVs still showing the capacitive behavior at the lower temperatures (Supplementary Fig. 15b). The shape of the CV curves became similar to what would be expected from a classic electrochemical double-layer capacitor as the redox/intercalation peaks disappeared. It is safe to assume that the double-layer component of the charge mechanism of these electrodes dominates at sub-zero temperatures.

Since electrochemical reactions are suppressed at low temperatures, a larger operating potential window should be possible at lower temperatures without the occurrence of any harmful side reactions (Fig. 4e). The highest cell voltage we achieved was $4.2 \mathrm{~V}$ with a coulombic efficiency of $97 \%$ at $-30{ }^{\circ} \mathrm{C}$, significantly larger than the voltage windows of previously reported asymmetric MXene-based supercapacitor systems. Moreover, the capacitance remained approximately the same when the voltage window was increased, leading to a significantly improved energy density at lower temperatures. Supplementary Fig. 17 showed the relationship between the energy and power densities normalized to the mass of both electrodes at low temperatures for the asymmetric cell. An impressive energy density of $59 \mathrm{Wh} \mathrm{kg}^{-1}$ and a power density of $9.6 \mathrm{~kW} \mathrm{~kg}^{-1}$ were obtained at $-30^{\circ} \mathrm{C}$, surpassing the best values reported for supercapacitors with $2 \mathrm{D}$ electrode materials operating at low temperatures ${ }^{35-40}$.

We compared the performance of our MXene-knotted CNT composite electrode with the electrodes based on commercial high surface area activated carbon (AC). The AC-based symmetric cell showed a capacitance of $24 \mathrm{~F} \mathrm{~g} \mathrm{~g}^{-1}$ at $10 \mathrm{mV} \mathrm{s}^{-1}$ and a capacitance retention of $29 \%$ from 10 to $500 \mathrm{mV} \mathrm{s}^{-1}$. In addition, the capacitance of the AC-based symmetric cell decreased from 23 to $1.5 \mathrm{~F} \mathrm{~g}^{-1}$ at $20 \mathrm{mV} \mathrm{s}^{-1}$ when the temperature decreased from 20 to $-60^{\circ} \mathrm{C}$ (Supplementary Fig. 18). As seen in Supplementary Fig. 17, our cell has a comparable capacitance of $\sim 23 \mathrm{~F} \mathrm{~g}^{-1}$ with the AC-based cell, yet has much improved capacitance retention at increased scan rates ( $50 \%$ from 10 to $500 \mathrm{mV} \mathrm{s}^{-1}$ ) and at low temperatures ( $55 \%$ from 20 to $-60^{\circ} \mathrm{C}$ ) (Fig. $4 \mathrm{f}$ ). Moreover, our cell had a larger voltage window $(4.2 \mathrm{~V})$ than that of the AC-based symmetric cells $(3 \mathrm{~V})$ at low temperatures, which delivered energy density of $59 \mathrm{Wh} \mathrm{kg}^{-1}$ that was almost four times the value of the AC-based cell $\left(\sim 15 \mathrm{Wh} \mathrm{kg}^{-1}\right)$.

The low-temperature performances of the asymmetric cells with the MXene-knotted CNT composite electrodes with different CNT contents are shown in Fig. $4 \mathrm{f}$ and Supplementary Fig. 19. Although the asymmetric cell with the $17 \%$ CNT electrode as the negative electrode had the highest capacitance of $20 \mathrm{~F} \mathrm{~g}^{-1}$ at low temperatures (Supplementary Fig. 19), the asymmetric cell with the $9 \%$ CNT electrode had the highest capacitance retention of $\sim 55 \%$ over the temperature range of 20 to $-60^{\circ} \mathrm{C}$. This result suggested that the electronic conductivity of the electrodes also played an important role during lowtemperature operation. The Nyquist plots of the cell with the $17 \%$ CNT electrode exhibited almost identical curves from 20 to $-60{ }^{\circ} \mathrm{C}$, indicating the temperature-independent capacitive behavior (Supplementary Fig. 20a). Conversely, the Nyquist plots of the asymmetric cell with pure $\mathrm{Ti}_{3} \mathrm{C}_{2}$ MXene as the negative electrode were severely distorted to the right at low temperatures (Supplementary Fig. 20b). This deviation from the ideal capacitive behavior matched the rapid decrease of capacitance at lower temperatures for the asymmetric cell with the pure $\mathrm{Ti}_{3} \mathrm{C}_{2}$ electrode (Fig. 4f). In addition, when the temperature decreased from 20 to $-60{ }^{\circ} \mathrm{C}$, the equivalent series resistance (ESR) values of the cell with the $17 \% \mathrm{CNT}$ electrode and the cell with the pure $\mathrm{Ti}_{3} \mathrm{C}_{2}$ film increased from 0.28 to 

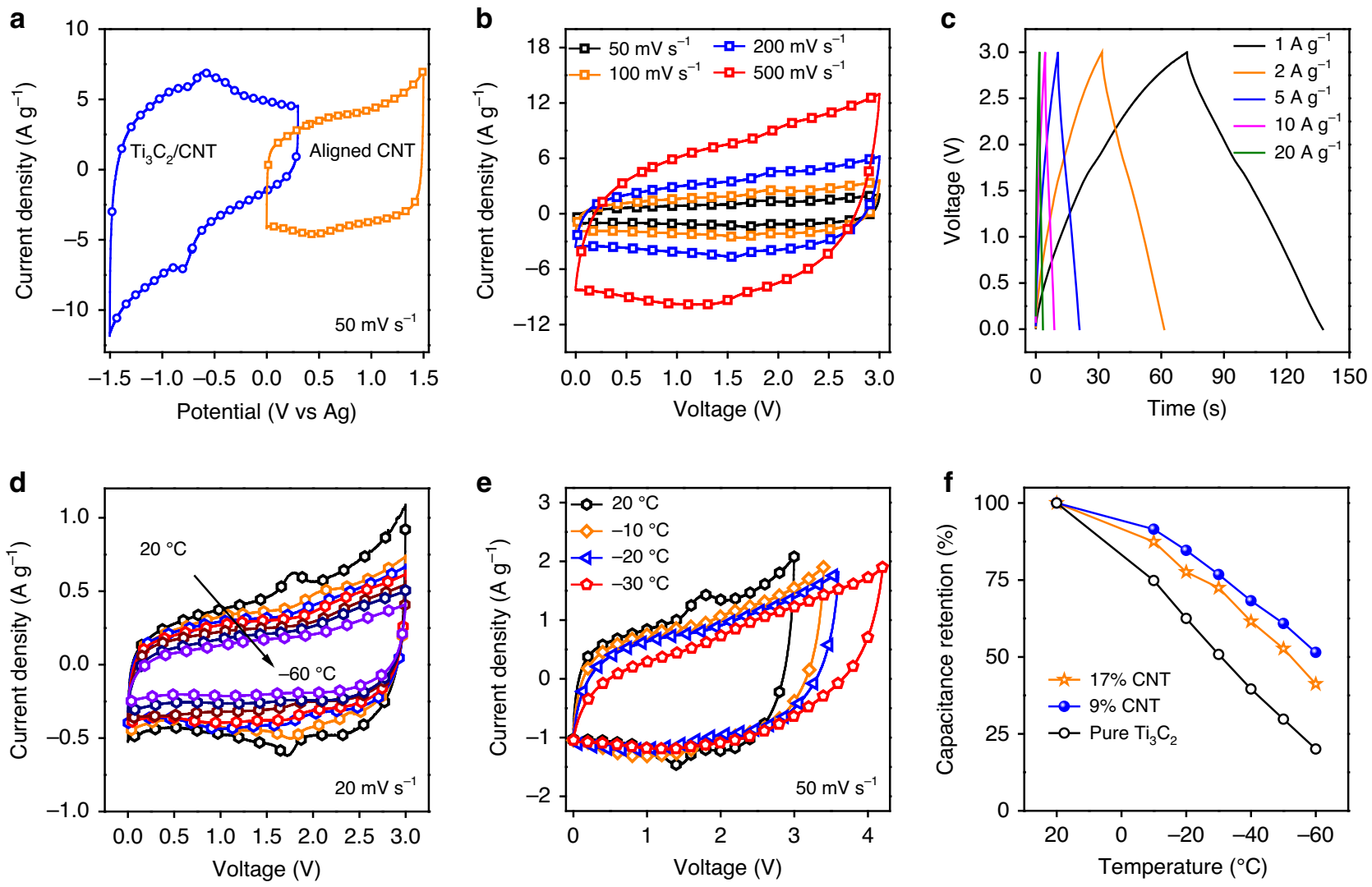

Fig. 4 Electrochemical tests for asymmetric full cells. a Full cell fabrication: three-electrode $\mathrm{CVs}$ of the MXene-knotted $\mathrm{CNT}$ ( $\mathrm{Ti}_{3} \mathrm{C}_{2} / \mathrm{CNT}_{\mathrm{C}}$ ) composite electrode and aligned CNT electrode at $50 \mathrm{mV} \mathrm{s}^{-1}$. b Cyclic voltammograms for the full cell at different scan rates. c Galvanostatic charge-discharge curves for the full cell at different current densities. d Cyclic voltammograms at different temperatures at $20 \mathrm{mV} \mathrm{s}^{-1}$ and e cyclic voltammograms with larger voltage windows at low temperatures for the full cell using MXene-knotted CNT composite electrode with a CNT content of $17 \%$ as the negative electrode. f Capacitance retention of full cells with different $\mathrm{Ti}_{3} \mathrm{C}_{2} \mathrm{MXene-based} \mathrm{electrodes} \mathrm{as} \mathrm{a} \mathrm{function} \mathrm{of} \mathrm{temperature.}$

$1.91 \Omega \mathrm{cm}^{2}$ and from 0.24 to $1.35 \Omega \mathrm{cm}^{2}$, respectively (Supplementary Fig. 20c, d). It can be inferred that the increase of ESR values was associated with the lowered ionic conductivity of the electrolyte at low temperatures (Supplementary Fig. 21).

Although the pure $\mathrm{Ti}_{3} \mathrm{C}_{2}$ electrode had higher conductivity, the MXene-knotted CNT composite electrodes performed better at lower temperatures. From these results, it is clear that the architecture and ionic conductivity of the electrode are key factors for developing capacitive energy storage systems capable of operating at low temperatures where electrolyte kinetics are limiting.

\section{Discussion}

We have demonstrated how the architecture of an electrode can lead to enhanced rate performances in organic electrolytes. By using specially designed knotted CNTs, the MXene-knotted CNT composite electrodes showed a high capacitance retention of $\sim 56 \%$ over a scan rate range of $10 \mathrm{mV} \mathrm{s}^{-1}$ to $10 \mathrm{~V} \mathrm{~s}^{-1}$ in a mixed organic electrolyte. The large knot-like structures in the knotted CNTs were essential for disrupting the alignment of the $\mathrm{Ti}_{3} \mathrm{C}_{2}$ flakes, leading to the ion transport pathways with lower tortuosity and enhanced ion accessibility. The advantages of the electrode resulted in improved low-temperature operation for a $\mathrm{Ti}_{3} \mathrm{C}_{2}$ MXene-based supercapacitor, with a high capacitance retention of $\sim 55 \%$ when the operating temperature was decreased from 20 to $-60^{\circ} \mathrm{C}$. This study has demonstrated that pseudocapacitive materials can be used for energy storage at high rates in organic electrolyte after tailoring the design of the electrode architecture.

\section{Methods}

Preparation of $\mathrm{Ti}_{3} \mathbf{C}_{\mathbf{2}}$ solution. Multilayer $\mathrm{Ti}_{3} \mathrm{C}_{2}$ was produced by etching $\mathrm{Ti}_{3} \mathrm{AlC}_{2}$ MAX phase (Carbon-Ukraine) in an acidic fluoride containing etchant ${ }^{41}$. One gram of $\mathrm{Ti}_{3} \mathrm{AlC}_{2}$ was slowly added to $20 \mathrm{~mL}$ of the etchant solution which contained $12 \mathrm{~mL}$ of $9 \mathrm{M} \mathrm{HCl}, 2 \mathrm{~mL}$ of $49 \% \mathrm{HF}$, and $6 \mathrm{~mL}$ of $\mathrm{H}_{2} \mathrm{O}$. The mixture was stirred at $35{ }^{\circ} \mathrm{C}$ for $24 \mathrm{~h}$ using a hot plate. The multilayer $\mathrm{Ti}_{3} \mathrm{C}_{2}$ was washed with deionized water by repeated centrifugation and decantation until the $\mathrm{pH}$ of the supernatant was neutral. The sediment was dispersed in $50 \mathrm{~mL}$ of deionized water and delaminated by adding $1 \mathrm{~g} \mathrm{LiCl}$. The solution was then stirred for $6 \mathrm{~h}$ and then washed for four times with deionized water by centrifuging at 3500 r.p.m. The obtained single layer $\mathrm{Ti}_{3} \mathrm{C}_{2}$ was then dispersed in deionized water and sonicated for $1 \mathrm{~h}$ with Ar bubbling in an ice bath to reduce the size of the flakes. After centrifuging of the sonicated solution at 3500 r.p.m. for $1 \mathrm{~h}$, the final $\mathrm{Ti}_{3} \mathrm{C}_{2}$ solution in water was collected as the dark supernatant.

Preparation of knotted CNTs. Ni-Mn-Al-O catalysts were synthesized using a typical co-precipitation and subsequent calcination reaction. First, $\mathrm{Ni}\left(\mathrm{NO}_{3}\right)_{3}, \mathrm{Mn}$ $\left(\mathrm{NO}_{3}\right)_{2}$, and $\mathrm{Al}\left(\mathrm{NO}_{3}\right)_{3}$ were dissolved in $200.0 \mathrm{~mL}$ deionized water with $\left[\mathrm{Ni}^{2+}\right]+$ $\left[\mathrm{Mn}^{2+}\right]+\left[\mathrm{Al}^{3+}\right]=0.2 \mathrm{~mol} \mathrm{~L}^{-1}, \mathrm{n}(\mathrm{Ni}): \mathrm{n}(\mathrm{Mn}): \mathrm{n}(\mathrm{Al})=0.4: 1: 2$. Then, $50 \mathrm{~mL} 2.5$ mol L ${ }^{-1} \mathrm{NaOH}$ was added into the solution and the solution was then left to stand at $95^{\circ} \mathrm{C}$ for $12 \mathrm{~h}$ in a $500 \mathrm{~mL}$ flask, which was equipped with a reflux condenser in ambient atmosphere. The as-obtained suspension was filtered, washed by deionized water, and freeze-dried to get the NiMnAl LDHs. The NiMnAl LDHs were then loaded into a quartz boat and calcinated at $700{ }^{\circ} \mathrm{C}$ for $2 \mathrm{~h}$ in a quartz tube to get the final product $\mathrm{Ni}-\mathrm{Mn}-\mathrm{Al}-\mathrm{O}$.

The knotted CNTs were prepared using a temperature shift two-stage fluidized bed. In detail, $0.2 \mathrm{~g} \mathrm{Ni}-\mathrm{Mn}-\mathrm{Al}-\mathrm{O}$ nanoparticles were loaded into the reactor and processed with our customed two-step growth (Supplementary Fig. 2). The first-step growth was held in the upper zone of the reactor with the temperature of $750{ }^{\circ} \mathrm{C}$. The nanoparticles were immersed in the $\mathrm{H}_{2}$ (flow rate of $80 \mathrm{~mL} \mathrm{~min}^{-1}$ ) for $10 \mathrm{~min}$ for the catalyst formation and then $\mathrm{C}_{2} \mathrm{H}_{2}$ (flow rate of $100 \mathrm{~mL} \mathrm{~min}^{-1}$ ) was introduced for growing CNTs for $15 \mathrm{~min}$. During the first-step growth, the large-sized catalysts were conceived from the nanoparticles and thick CNTs were acquired with the average diameter of $\sim 55 \mathrm{~nm}$ (Supplementary Fig. 3a). Then the isolation board was opened and the nanoparticles (with the thick CNTs on them) were moved to the lower zone 
of the reactor with the temperature of $950^{\circ} \mathrm{C}$ to proceed the second-step growth for another $15 \mathrm{~min}$. During this process, the small catalysts were conceived from the nanoparticles and the thin CNTs were grown with the average diameter of $\sim 15 \mathrm{~nm}$ (Supplementary Fig. 3b). Then the knotted structured CNTs with a bimodal diameter distribution were obtained. The furnace was cooled to room temperature under Ar protection. The as-grown products were collected and purified by routine $\mathrm{HCl}$ $\left(5 \mathrm{~mol} \mathrm{~L}^{-1}\right)$ and $\mathrm{NaOH}\left(13 \mathrm{~mol} \mathrm{~L}^{-1}\right)$ treatment.

Preparation of electrodes. For the preparation of MXene-knotted CNT composite electrodes, the knotted CNTs were first dispersed in a CTAB solution (0.1 wt\%) by probe sonication to get a CNT concentration of $0.5 \mathrm{mg} \mathrm{mL}^{-1}$. MXene-knotted CNT composite electrodes were prepared by a self-assembly process. Typically, a CTAB-grafted CNT solution was added dropwise to a $\mathrm{Ti}_{3} \mathrm{C}_{2}$ MXene suspension with varying $\mathrm{CNT}$ contents from $5 \%$ to $34 \%$. The mixture was probe sonicated for $10 \mathrm{~min}$ and then filtered using a Celgard membrane (3501). MXene-knotted CNT composite electrodes were obtained after washing with deionized water and vacuum drying at $80^{\circ} \mathrm{C}$

For comparison, MXene-MWCNT composite electrodes were prepared by using the same self-assembly method except for using the commercially available nonknotted MWCNTs (Shenzhen NANO Tech. Port. Co. Ltd, China). The AC electrodes were prepared by mixing $80 \mathrm{wt} \%$ AC (YP50, Kuraray chemical, Japan), $10 \mathrm{wt} \%$ acetylene black (Alfa Aesar, 99.9\%), and $10 \mathrm{wt} \%$ poly(tetrafluoroethylene) (PTFE, Aladdin, $60 \%$ ) as a binder in ethanol. The solution was stirring overnight, and ethanol was then evaporated at $80^{\circ} \mathrm{C}$, forming a rubber-like paste. The paste was rolled into a film for use. The mass loading of the AC electrode was $\sim 2 \mathrm{mg} \mathrm{cm}^{-2}$.

The aligned CNT electrode was prepared by directly using the aligned CNT forest that was synthesized by the water-assisted CVD method as the positive electrodes. For the synthesis of the aligned CNT forest, $\mathrm{Al}_{2} \mathrm{O}_{3}(\sim 30 \mathrm{~nm}) / \mathrm{Fe}(\sim 2 \mathrm{~nm})$ metal layers were firstly sputtered on an Si substrate with an oxide layer $(600 \mathrm{~nm})$. Then, the catalyst coated substrate was inserted into a quartz tube furnace under flowing $\mathrm{Ar}$ and $\mathrm{H}_{2}$ (total flow $1000 \mathrm{~mL} \mathrm{~min}-1$ ). When reaching the reaction temperature of $750^{\circ} \mathrm{C}, \mathrm{C}_{2} \mathrm{H}_{4}$ was introduced into the furnace with a flow rate of $100 \mathrm{~mL} \mathrm{~min}{ }^{-1}$. The CVD growth was maintained for $15 \mathrm{~min}$ at $750^{\circ} \mathrm{C}$ with a water vapor concentration of $\sim 75 \mathrm{ppm}$.

Material characterization. The morphology of $\mathrm{Ti}_{3} \mathrm{C}_{2}$ MXene and MXene-knotted CNT composite electrodes were observed using SEM (Zeiss Surra 50VP, Germany or FE-SEM, FEI Nova 450 Nano) and TEM (JEOL JEM-2100, Japan). Structure of the $\mathrm{Ti}_{3} \mathrm{C}_{2}$ MXene and MXene-knotted CNT composite electrodes were characterized by XRD (SmartLab, Rigaku 112 Corp. or X'Pert Pro, PANanalytical) using $\mathrm{Cu} \mathrm{Ka}{ }_{1}$ radiation. Surface chemistry of the MXene-knotted CNT composite electrodes was characterized by XPS (ESCALab 250).

Electrochemical tests. Electrochemical measurements were performed in threeelectrode Swagelok-type cells. Pure $\mathrm{Ti}_{3} \mathrm{C}_{2}$ MXene electrodes prepared by vacuum filtration and MXene-knotted CNT composite electrodes were used directly as the working electrodes and AC films (YP50, Kuraray) with 5\% PTFE were used as the counter electrodes. A silver wire was used as the reference electrode. Polypropylene membranes (Celgard 3501) were used as the separators, and $1 \mathrm{M}$ solution of EMIM-TFSI/Li-TFSI (EMIM-TFSI: Li-TFSI $=1: 1$ ) in ACN or $1 \mathrm{M}$ EMIM-TFSI/ ACN was used as the electrolyte. For the asymmetric cells, aligned CNTs were chosen as the positive electrode and assembled with the MXene-knotted CNT composite electrodes in the Swagelok-type cells. Electrochemical measurements of GCD, cyclic voltammetry and EIS were performed using a VMP3 potentiostat (BioLogic). Scanning rates ranging from $10 \mathrm{mV} \mathrm{s}^{-1}$ to $10 \mathrm{~V} \mathrm{~s}^{-1}$ were used for the cyclic voltammetry experiments with a working potential window of $1.8 \mathrm{~V}(-1.5$ to $0.3 \mathrm{~V}$ versus $\mathrm{Ag}$ ). The impedance spectroscopy analysis was performed from $10 \mathrm{mHz}$ to $200 \mathrm{kHz}$ from OCV to the maximum applied voltage. Cycling stability was tested at a scan rate of $100 \mathrm{mV} \mathrm{s}^{-1}$ for 10,000 cycles. The low-temperature tests were conducted in a high/low-temperature test chamber (MCB-1.2-.33-.33-H/AC). The ionic conductivity of the electrolyte at different temperature was tested by using the conductivity isopod (EDAQ/EPU357).

Calculations for the electrochemical tests. The capacitance of a single electrode $\left(C_{\text {sp }}\right)$ in the three-electrode cell was calculated from the anodic scan of $\mathrm{CV}$ curve based on

$$
C_{\mathrm{sp}}=\frac{\int i \cdot \mathrm{d} t}{m \cdot V},
$$

where $i$ was the discharging current, $m$ was the mass of the working electrode and $V$ was the voltage window $(1.8 \mathrm{~V})$ of the $\mathrm{CV}$ scan. $C_{\mathrm{sp}}$ was also calculated from the discharging curve of GCD for comparison.

For the two-electrode cell, the capacitance of the full cell $\left(C_{\text {cell }}\right)$ was calculated from the cathodic scan of the $\mathrm{CV}$ curves:

$$
C_{\text {cell }}=\frac{\int I \cdot \mathrm{d} t}{m_{\text {total }} \cdot V_{\text {cell }}},
$$

where $m_{\text {total }}$ was the whole mass of both electrodes and $V_{\text {cell }}$ was the voltage window of the full cell.
The energy density of the full cell was estimated based on the discharging curves of the GCD:

$$
E_{\text {cell }}=I\left(\int_{0}^{t_{\mathrm{d}}} V \mathrm{~d} t\right),
$$

where $I$ was the discharging current density, $t_{\mathrm{d}}$ was the discharging time and $V$ was the voltage of the asymmetric cell. The power density was further evaluated based on $P=E_{\text {cell }} / \Delta t$.

\section{Data availability}

The data that support the plots within this paper and other finding of this study are available from the corresponding author upon reasonable request.

Received: 4 May 2020; Accepted: 4 November 2020; Published online: 02 December 2020

\section{References}

1. Simon, P. \& Gogotsi, Y. Capacitive energy storage in nanostructured carbonelectrolyte systems. Acc. Chem. Res. 46, 1094-1103 (2013).

2. Salanne, M. et al. Efficient storage mechanisms for building better supercapacitors. Nat. Energy 1, 16070 (2016).

3. Beguin, F., Presser, V., Balducci, A. \& Frackowiak, E. Carbons and electrolytes for advanced supercapacitors. Adv. Mater. 26, 2219-2251 (2014).

4. Wang, X. et al. Pseudocapacitance of MXene nanosheets for high-power sodium-ion hybrid capacitors. Nat. Commun. 6, 6544 (2015).

5. Choi, C. et al. Achieving high energy density and high power density with pseudocapacitive materials. Nat. Rev. Mater. 5, 5-19 (2019).

6. Augustyn, V., Simon, P. \& Dunn, B. Pseudocapacitive oxide materials for high-rate electrochemical energy storage. Energy Environ. Sci. 7, 1597 (2014).

7. Augustyn, V. et al. High-rate electrochemical energy storage through Li+ intercalation pseudocapacitance. Nat. Mater. 12, 518-522 (2013).

8. Wang, X. et al. Influences from solvents on charge storage in titanium carbide MXenes. Nat. Energy 4, 241-248 (2019).

9. $\mathrm{Xu}$, J. et al. Temperature-independent capacitance of carbon-based supercapacitor from -100 to $60^{\circ} \mathrm{C}$. Energy Storage Mater. 22, 323-329 (2019).

10. Jin, T., Li, H., Li, Y., Jiao, L. \& Chen, J. Intercalation pseudocapacitance in flexible and self-standing $\mathrm{V}_{2} \mathrm{O}_{3}$ porous nanofibers for high-rate and ultrastable K ion storage. Nano Energy 50, 462-467 (2018).

11. Ren, C. E. et al. Porous two-dimensional transition metal carbide (MXene) flakes for high-performance Li-ion storage. ChemElectroChem 3, 689-693 (2016).

12. Kajiyama, S. et al. Enhanced Li-ion accessibility in MXene titanium carbide by steric chloride termination. Adv. Energy Mater. 7, 1601873 (2017).

13. Xie, X. et al. Porous heterostructured MXene/carbon nanotube composite paper with high volumetric capacity for sodium-based energy storage devices. Nano Energy 26, 513-523 (2016).

14. Zhao, M. Q. et al. Flexible MXene/carbon nanotube composite paper with high volumetric capacitance. Adv. Mater. 27, 339-345 (2015).

15. Luo, J. et al. Pillared structure design of MXene with ultralarge interlayer spacing for high-performance lithium-ion capacitors. ACS Nano 11, 2459-2469 (2017).

16. Dall'Agnese, Y., Rozier, P., Taberna, P.-L., Gogotsi, Y. \& Simon, P. Capacitance of two-dimensional titanium carbide (MXene) and MXene/ carbon nanotube composites in organic electrolytes. J. Power Sources $\mathbf{3 0 6}$ 510-515 (2016)

17. Sun, H. et al. Three-dimensional holey-graphene/niobia composite architectures for ultrahigh-rate energy storage. Science 356, 599-604 (2017)

18. Anasori, B., Lukatskaya, M. R. \& Gogotsi, Y. 2D metal carbides and nitrides (MXenes) for energy storage. Nat. Rev. Mater. 2, 16098 (2017).

19. Naguib, M., Mochalin, V. N., Barsoum, M. W. \& Gogotsi, Y. 25th anniversary article: MXenes: a new family of two-dimensional materials. Adv. Mater. 26, 992-1005 (2014).

20. Lukatskaya, M. R. et al. Cation intercalation and high volumetric capacitance of two-dimensional titanium carbide. Science 341, 1502-1505 (2013).

21. Lukatskaya, M. R. et al. Ultra-high-rate pseudocapacitive energy storage in two-dimensional transition metal carbides. Nat. Energy 2, 17105 (2017).

22. Xia, Y. et al. Thickness-independent capacitance of vertically aligned liquidcrystalline MXenes. Nature 557, 409-412 (2018).

23. Xu, M., Futaba, D. N., Yumura, M. \& Hata, K. Alignment control of carbon nanotube forest from random to nearly perfectly aligned by utilizing the crowding effect. ACS Nano 6, 5837-5844 (2012).

24. Xu, M., Du, F., Ganguli, S., Roy, A. \& Dai, L. Carbon nanotube dry adhesives with temperature-enhanced adhesion over a large temperature range. Nat. Commun. 7, 13450 (2016) 
25. Futaba, D. N. et al. Shape-engineerable and highly densely packed singlewalled carbon nanotubes and their application as super-capacitor electrodes. Nat. Mater. 5, 987-994 (2006).

26. Xu, M., Futaba, D. N., Yamada, T., Yumura, M. \& Hata, K. Carbon nanotubes with temperature-invariant viscoelasticity from $-196^{\circ}$ to $1000^{\circ} \mathrm{C}$. Science $\mathbf{3 3 0}$, 1364-1368 (2010).

27. Lu, M. et al. Tent-pitching-inspired high-valence period 3-cation preintercalation excels for anode of 2D titanium carbide (MXene) with high $\mathrm{Li}$ storage capacity. Energy Storage Mater. 16, 163-168 (2019).

28. Liu, Y. T. et al. Self-assembly of transition metal oxide nanostructures on MXene nanosheets for fast and stable lithium storage. Adv. Mater. 30, 1707334 (2018).

29. Korenblit, Y. et al. In situ studies of ion transport in microporous supercapacitor electrodes at ultralow temperatures. Adv. Funct. Mater. 22, 1655-1662 (2012).

30. Lin, R. et al. Capacitive energy storage from -50 to $100{ }^{\circ} \mathrm{C}$ using an ionic liquid electrolyte. J. Phys. Chem. Lett. 2, 2396-2401 (2011).

31. Taberna, P. L., Simon, P. \& Fauvarque, J. F. Electrochemical characteristics and impedance spectroscopy studies of carbon-carbon supercapacitors. $J$. Electrochem. Soc. 150, A292 (2003).

32. Segalini, J., Daffos, B., Taberna, P. L., Gogotsi, Y. \& Simon, P. Qualitative electrochemical impedance spectroscopy study of ion transport into subnanometer carbon pores in electrochemical double layer capacitor electrodes. Electrochim. Acta 55, 7489-7494 (2010).

33. Simon, P., Gogotsi, Y. \& Dunn, B. Where do batteries end and supercapacitors begin? Science 343, 1210-1211 (2014).

34. Gogotsi, Y. \& Penner, R. M. Energy storage in nanomaterials-capacitive, pseudocapacitive, or battery-like? ACS Nano 12, 2081-2083 (2018).

35. Tsai, W.-Y. et al. Outstanding performance of activated graphene based supercapacitors in ionic liquid electrolyte from -50 to $80^{\circ} \mathrm{C}$. Nano Energy 2 , 403-411 (2013).

36. Lin, Z., Taberna, P.-L. \& Simon, P. Graphene-based supercapacitors using eutectic ionic liquid mixture electrolyte. Electrochim. Acta 206, 446-451 (2016).

37. Liu, W., Yan, X., Lang, J. \& Xue, Q. Effects of concentration and temperature of EMIMBF4/acetonitrile electrolyte on the supercapacitive behavior of graphene nanosheets. J. Mater. Chem. 22, 8853 (2012).

38. Zhou, Y. et al. High performance supercapacitor under extremely low environmental temperature. RSC Adv. 5, 71699-71703 (2015).

39. Tian, J. et al. EMIMBF4-GBL binary electrolyte working at $-70^{\circ} \mathrm{C}$ and $3.7 \mathrm{~V}$ for a high performance graphene-based capacitor. J. Mater. Chem. A $\mathbf{6}$, 3593-3601 (2018)

40. Vellacheri, R. et al. High performance supercapacitor for efficient energy storage under extreme environmental temperatures. Nano Energy 8, 231-237 (2014).

41. Levitt, A. S. et al. Electrospun MXene/carbon nanofibers as supercapacitor electrodes. J. Mater. Chem. A 7, 269-277 (2019).

\section{Acknowledgements}

The authors acknowledge the financial support from National Natural Science Foundation of China (Grant No. 51972127), Applied Basic Research Programs of Wuhan City (Grant No. 2018010401011282), Fundamental Research Project of Shenzhen
(Grant No. JCYJ20190809102607400), and Natural Science Foundation of Hubei Province, China (Grant No. 2018CFA049). X.G. specially acknowledges the support by the scholarship from Huazhong University of Science and Technology (HUST). The authors thanked the Analytical and Testing Center of Huazhong University of Science and Technology for the assistance on sample characterization. The work of T.S.M., X.W., and Y.G. on MXene synthesis and electrochemical characterization was supported by the Fluid Interface Reactions, Structures and Transport (FIRST) Center, an Energy Frontier Research Center (EFRC) funded by the U.S. Department of Energy, Office of Science, and Office of Basic Energy Sciences.

\section{Author contributions}

M.X., Y.G., and X.G. conceived the study and planned the experiments. M.X., X.D. and J.S. synthesized the knotted CNTs. T.S.M. synthesized MXene flakes. X.G. fabricated MXene-knotted CNT composite electrodes and conducted electrochemical testing. X.W. suggested on the low-temperature electrolyte. X.G., M.Z., T.S.M. and J.S. performed structural characterization. M.X., Y.G. and X.G. prepared the manuscript. All the authors discussed the results and commented on the manuscript at all stages.

\section{Competing interests}

The authors declare no competing interests.

\section{Additional information}

Supplementary information is available for this paper at https://doi.org/10.1038/s41467020-19992-3.

Correspondence and requests for materials should be addressed to Y.G. or M.X.

Peer review information Nature Communications thanks Andrews Grace, Qing Huang and the other, anonymous, reviewer(s) for their contribution to the peer review of this work. Peer reviewer reports are available.

Reprints and permission information is available at http://www.nature.com/reprints

Publisher's note Springer Nature remains neutral with regard to jurisdictional claims in published maps and institutional affiliations.

pen Access This article is licensed under a Creative Common Attribution 4.0 International License, which permits use, sharing adaptation, distribution and reproduction in any medium or format, as long as you give appropriate credit to the original author(s) and the source, provide a link to the Creative Commons license, and indicate if changes were made. The images or other third party material in this article are included in the article's Creative Commons license, unless indicated otherwise in a credit line to the material. If material is not included in the article's Creative Commons license and your intended use is not permitted by statutory regulation or exceeds the permitted use, you will need to obtain permission directly from the copyright holder. To view a copy of this license, visit http://creativecommons.org/ licenses/by/4.0/

(c) The Author(s) 2020 